\title{
Las píldoras de aprendizaje como metodología para afianzar conceptos en alumnos de Máster de Arquitectura y edificación
}

\author{
F. Javier Cárcel-Carrasco ${ }^{\mathrm{a}}$, Elisa Peñalvo-López \\ ${ }^{a}$ Universitat Politècnica de València. Email: fracarc1@csa.upv.es \\ ${ }^{\mathrm{b}}$ Universitat Politècnica de València. Email: elpealpe@upvnet.upv.es
}

\begin{abstract}
Resumen
Definiendo los Estilos de Aprendizaje como las estrategias por las cuales se procesa la información en su proceso de aprendizaje, dichos estilos varían con la formación, edad, las materias, el nivel de éxito, la cultura y el género, que pueden ser modificados. Por ello, los docentes deben mostrar interés por descubrir el estilo del alumnado tipo al que va dedicada la enseñanza y adaptarlo a las características de cada situación. Teniendo en cuenta dichas características, en este artículo, se muestran las experiencias realizadas mediante los conceptos de pildoras de aprendizaje (o conceptos básicos), como forma de posicionar conocimientos para estructurar una asignatura de máster oficial en Arquitectura y Edificación, en las primeras horas docentes de comienzo de dichas asignaturas y la visión y percepción de los estudiantes.
\end{abstract}

Palabras clave: Estilo de aprendizaje; formación postgrado; ingenieros edificación, Arquitectos, píldoras de aprendizaje.

\section{Introducción}

Existen diferentes teorías sobre estilos de aprendizaje (Alonso et al., 1994; 1999; 2005) que explican porqué cada persona puede aprender de diferente manera afectando a su rendimiento, para lo cual es necesario investigar y analizar los estilos de aprendizaje que comporten diferentes herramientas para la enseñanza que actuen con mayor eficiencia según los estilos.

Los estudios realizados han determinando cuatro estilos predominantes, el estilo activo, el reflexivo, el teórico y el pragmático. Una de las mejores definiciones fue realizada en los estudios de Alonso (Alonso et al., 1999): 
Las pildoras de aprendizaje como metodología para afianzar conceptos en alumnos de Máster de Arquitectura y edificación

- Activo. Se caracteriza por personas abiertas, entusiastas, que se lanzan hacia las nuevas experiencias.

- Reflexivo. Son individuos observadores y analíticos.

- Teórico. Tienen pensamiento lógico e integran sus observaciones dentro de teorías lógicas y complejas.

- Pragmático. Son personas muy basadas en su experiencia que intentan poner en práctica las ideas.

En un estudio realizado entre estudiantes de máster de arquitectura y edificación (Cárcel et al., 2016), se evidencia la tendencia particular por los estilos de aprendizaje reflexivo y teórico, aunque con poca diferencia entre ellos. Una extrategia para mejorar el aprendizaje entre este tipo de alumnos, podría ser el uso de pildoras de aprendizaje para afianzar y reforzar conocimiento y la inquietud por aprender del alumno.

Pueden considerarse las píldoras de aprendizaje como pequeñas piezas de material didáctico, formadas como objetos de aprendizaje de contenido audiovisual (Gallardo, 2015), diseñadas para sintetizar contenidos fundamentales de la asignatura, complementando las estrategias tradicionales de formación y facilitar la comprensión de algunos aspectos de la asignatura que presentan una mayor dificultad de comprensión para los estudiantes (Bengochea, 2011; Juanes et al., 2012; Maceiras et al, 2010). En estudios realizados, como el de Bengochea (Bengoechea 2011), constituyen un recurso de aprendizaje con gran atractivo para los jóvenes estudiantes, familiarizados con el formato audiovisual, similar al utilizado en YouTube.

En este artículo se estudia la percepción por parte de alumnos de máster de arquitectura y edificación, del uso de pildoras de aprendizaje en formato audiovisual, asi como pequeñas capsulas realizadas in-situ por el professor al comienzo de una area temática dentro del temario de una asignatura. Para ello se realizaron diferentes entrevistas al alumnado involucrado, para poder establecer el grado de aceptación y motivación.

\section{Estilos de aprendizaje y pildoras de conocimiento}

Las estrategias para formación es preciso analizar los procesos de aprendizaje hacia las peronas que está dirigida. Los Estilos de Aprendizaje se caracterizan por ser las estrategias por las cuales se procesa la información en su proceso contínuo de aprendizaje (Gravini, 2007) Varían con la formación de la persona, la profesión, el género, su edad y pueden variar en function de la experiencia o diversos aspectos. Es por ello que al elaborar una estrategia de formación, se tenga en cuenta los diferentes estilos de las personas que van a formar parte en la formación, para adaptarlo correctamente (Bahamón, 2006). 
En la década de los años 70, se empieza a mostrar interés por los estilos de aprendizaje por parte de Honey y Mumford, conceptualizándose después por Alonso, Gallego y Honey (Alonso et al., 1999), definiendo cuatro estilos de aprendizajes: activo, reflexivo, teórico y pragmático, caracterizandose por:

- Activo: Se involucra en experiencias nuevas, suele ser entusiasta ante lo novedoso y disfruta del momento presente, dejándose llevar por los acontecimientos lo que le lleva a actuar primero y a pensar después. Le aburre ocuparse de actividades a largo plazo y le gusta trabajar rodeados de gente, pero siendo el centro de éstas.

- Reflexivo: Analiza sus experiencias desde diversos prismas, profundizando en ellas hasta llegar a una conclusión. Observa y escucha antes de hablar. Para él lo más importante es recoger la información para su posterior análisis. Procura posponer las conclusiones.

- Teórico: adapta e integra sus observaciones en teorías complejas. Su pensamiento es secuencial integrando toda la información. Se siente incómodo con los juicios subjetivos y con las actividades ilógicas.

- Pragmático: Prueba ideas, teorías, técnicas y comprueba que funcionan en la práctica inmediatamente. Le impacientan las redundancias en la misma idea. Es práctico y cercano a la realidad al que le gusta tomar decisiones y resolver problemas, siendo éstos un desafío. Siempre busca la mejor manera de hacer las cosas.

Los resultados presentados en el estudio de Cárcel (Cárcel et al., 2016; Cárcel et al., 2014a; 2014b) permitieron caracterizar el perfil de aprendizaje de los titulados en ingeniería de la edificación para la muestra analizada de 125 personas. En la gráfica 1 se muestran los valores obtenidos, posicionandose en los ejes polares de cada tendencia para visionarlo de una manera gráfica, indicando que el predominante es un estilo reflexivo y teórico. 
Las pildoras de aprendizaje como metodología para afianzar conceptos en alumnos de Máster de Arquitectura y edificación

Gráfica 1: Gráfica polar con las medias absolutas de los estilos de aprendizaje

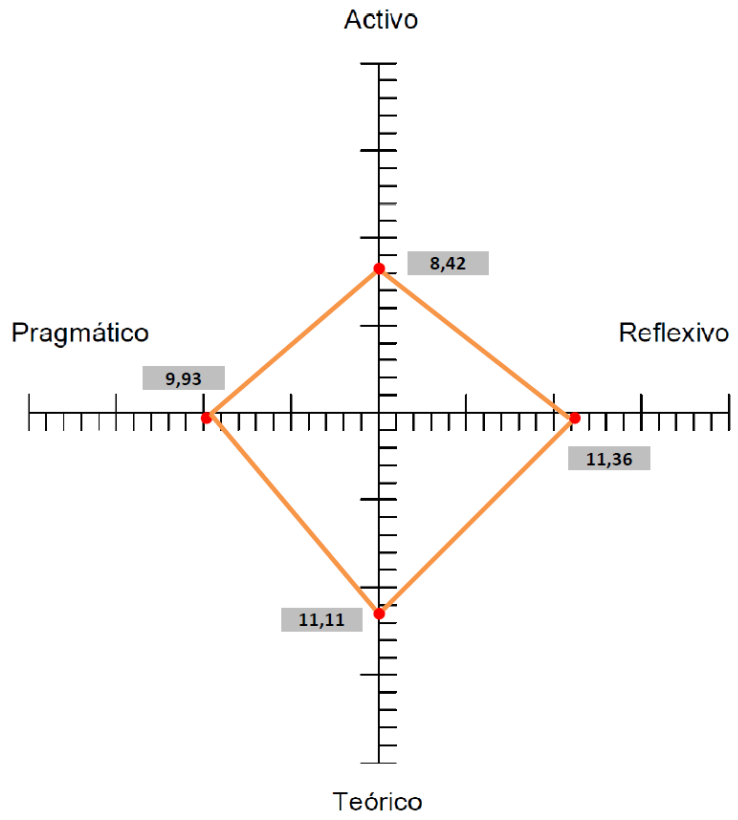

Fuente: Cárcel et al., 2016

En el estilo predominante reflexivo, se trata de personas analíticas. Prefieren pensar detenidamente sobre el objeto de estudio y trabajar solos. Aumentan la comprensión en pasos lineales, pueden no entender el material, pero logran conectar lógicamente sus partes.

En el estilo teórico, se trata de personas metódicas y con criterios objetivos, con un profundo sentido crítico, metódico y disciplinado, que abordan los problemas desde un punto de vista lógico. Prefieren las actividades estructuradas que les permitan comprender sistemas complejos.

Estas características definidas pueden ayudar a los docentes de estos titulados ante estudios post-grado a definir los modelos de enseñanza a aplicar a este tipo de estudiantes de arquitectura y edificación.

Que una persona tenga un estilo de aprendizaje reflexivo nos indica:

- Capacitados para recoger datos y analizarlos con detenimiento antes de llegar a una conclusión.

- Prefieren considerar todas las alternativas posibles antes de actuar.

- No les gusta intervenir hasta que consideran dominada la situación.

- Estudiantes ponderados, receptivos, analíticos y exhaustivos.

- Observadores, pacientes, cuidadosos y lentos en su proceso de aprendizaje. 
Ante personas con estilos de aprendizaje teóricos nos aporta:

- Tienden a ser perfeccionistas en la clase, lo que les inhibe en el momento de participar.

- Integran los hechos en teorías coherentes.

- Sienten preferencia por analizar y sintetizar lo aprendido.

Una Buena herramienta para la formación de este tipo de estudiantes sería mediante la utilización de pildoras de aprendizaje. Mediante esta herramienta, intentamos introducir al estudiante en una materia, afianzamos conceptos y abrimos la capacidad del alumno a entrar en temas más complejos. Una Pildora de aprendizaje o conocimiento es una unidad de conocimiento empaquetado en formato multimedia y puesto a disposicion para todo el mundo que lo necesite, aunque en este caso también llamamos así a la realizada por el professor antes de el comienzo de una material que necesite numerosas horas de docencia.

\section{Pildoras de aprendizaje}

La idea de las pildoras de aprendizaje es que su contenido puede ser utilizado y reutilizado desde un sistema de gestión de contenidos, como pueden ser los polimedias de la Universitat Politècnica de València (España), donde usualmente en esos videos de aprendizaje está la presencia de la imagen del docente. El tiempo que se considera apropiado para que un usuario comprenda un concepto, suele ser entre los 5 y los 10 minutos. Este material es útil tanto para ayudar a comprender en profundidad un problema (aprendizaje), y para aplicar el conocimiento a la resolución de nuevos problemas (habilidades) (Bengochea et al., 2012).

El sistema polimedia es un sistema diseñado en la Universitat Politècnica de València (España) en el año 2007, para la creación de contenidos multimedia para la educación presencial (semi-presencial), a distancia y/o virtual, que abarca desde la preparación del material docente hasta su distribución a través de distintos medios al publico o estudiantes objetivo (ICE, 2007; UPV, 2015).

La Pildora de Conocimiento es una pequena unidad de conocimiento explicito en un formato multimedia para ser utilizado desde la perspectiva formativa del "just-in-time" .

Para que cumplan su mision, las pildoras de aprendizaje deben tener las siguientes caracteristicas (Carrera, 2012):

- ser accesible para aquellos a los que se destina,

- $\quad$ ser accesible en el momento y lugar que sea. 
Las pildoras de aprendizaje como metodología para afianzar conceptos en alumnos de Máster de Arquitectura y edificación

\section{Elementos clave de las píldoras aprendizaje}

Una Pildora de aprendizaje es una unidad de conocimiento en formato multimedia y puesto a disposicion para todo el mundo que lo necesite; con algunos elementos clave que deben ser respetados (Carrera, 2012):

- $\quad$ el uso del formato multimedia,

- $\quad$ simplicidad, facilidad de crear y de distribuir,

- $\quad$ ser concise y breve,

- el profesor que tiene el conocimiento, deberia estar directamente involucrado,

Hay varios posibles formatos para las Pildoras del conocimiento:

- audio,

- video,

- presentacion multimedia.

La eleccion de un formato dependera siempre de:

- los objetivos del aprendizaje,

- el perfil de los alumnos o participantes,

- $\quad$ el momento en el cual los alumnos estan mas dispuestos a usar las Pildoras del Conocimiento,

- $\quad$ el equipo disponible para el uso de las Pildoras del Conocimiento.

El tipico contenido de una Pildora del Conocimiento esta compuesto por las siguientes partes (Carrera, 2012):

- $\quad$ título - un titulo que debera claramente identificar el objetivo de la Pildora del Conocimiento de modo que quien lo lea entienda inmediatamente si este es el contenido de lo que se necesita,

- una pregunta - una pregunta para responder que se puede introducir por via oral o en forma escrita, y en algunos formatos es conveniente presentar bajo las dos formas,

- una respuesta - la persona que tiene el conocimiento responde a la cuestion consultada en una forma clara, directa y breve,

- una lista de créditos - se cita aqui la informacion relevante sobre quien produjo y apoyo la creacion de la pildora del Conocimiento,

- palabras clave que permitirán una mejor busqueda de este tipo de contenido multimedia. 
En la figura 1 se muestran diferentes imágenes de polimedias grabados para utilización como píldoras de conocimiento entre alumnos del máster de arquitectura.
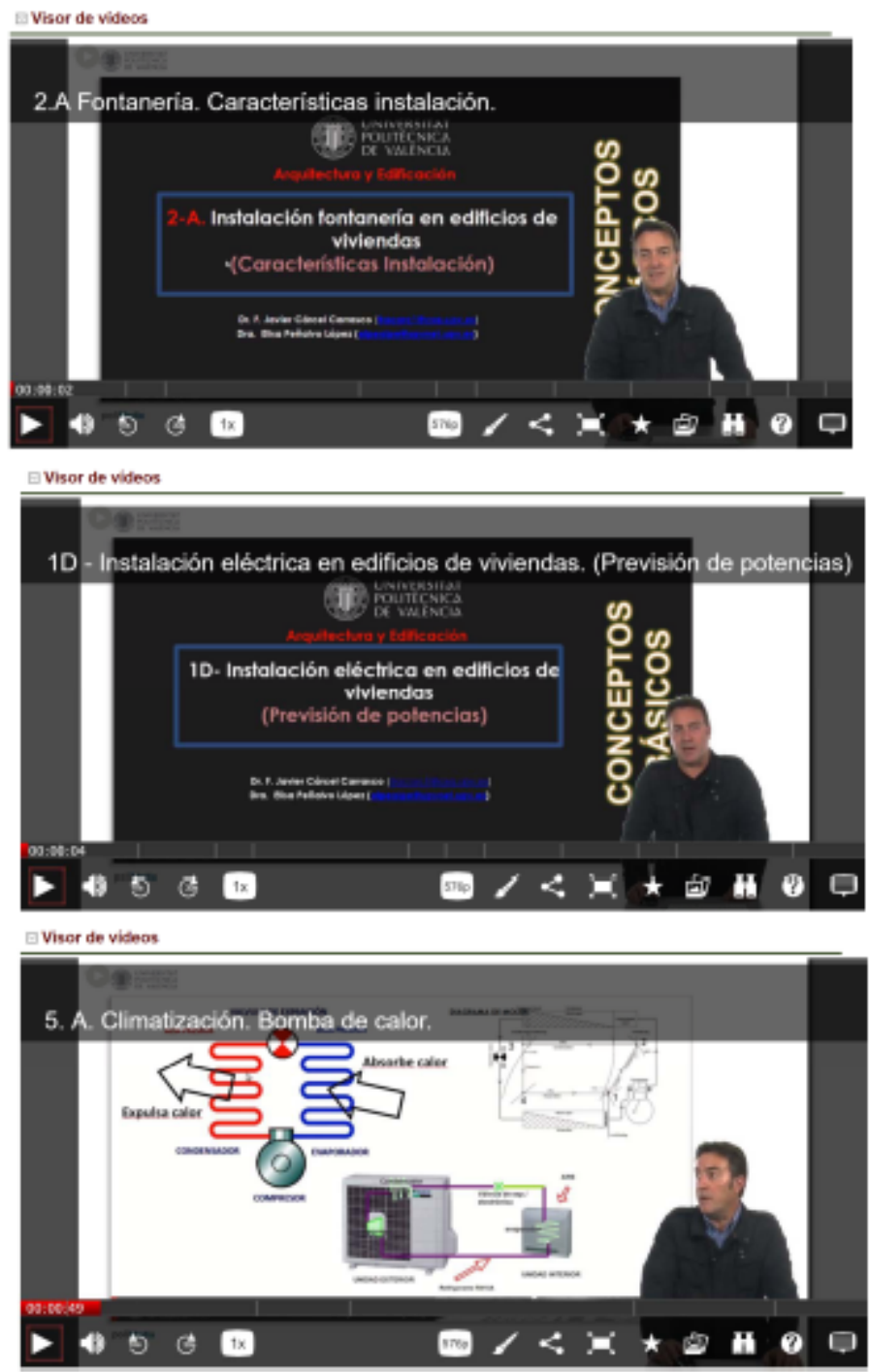

Fig. 1. Ejemplos de píldoras de conocimiento a través del sistema PoliMedia UPV

\section{Aplicación a estudiantes de máster de arquitectura y edificación}

Para estudiar la percepción de las píldoras de aprendizaje entre alumnos de máster de arquitectura, se realizaron diferentes polimedias sobre conceptos clave en referencia a 
Las pildoras de aprendizaje como metodología para afianzar conceptos en alumnos de Máster de Arquitectura y edificación

materias de instalaciones en la edificación. Al comienzo de un tema específico de instalaciones, el professor, in-situ, hacía un resumen clve del tema y explicaba la posición de la pildora de aprendizaje para que posteriormente el alumno pudiera visionarlas, acumular y asentar conocimientos, y obtener sus propias conclusiones.

Al final del curso, se realizó una entrevista corta en formato abierto a 40 alumnos (de entre 130 en total) de diferentes grupos, para conocer su percepción de la utilización de las pildoras de conocimiento. Con el fin de obtener información que no esté condicionada a las respuestas de los entrevistados, se sigue un protocolo de entrevista en profundidad semiestructurada con un estilo flexible, para extraer y entender las experiencias desde la visión del entrevistado (Cárcel et al., 2013).

Se realizaron cuatro preguntas cortas, que se indican a continuación, de las que se extrayeron las percepciones mayoritarias por parte de los alumnos:

1-¿Cúal es tu percepción en el uso de los polimedias como pildoras de conocimiento para afianzar los conceptos?.

De esta pregunta se extrae que la percepción mayoritaria de los alumnos ha sido positiva o muy positiva, dando la evidencia que la totalidad de los alumnos visualize los polimedias referenciados, visionandolos algunos de los alumnos varies veces a lo largo del curso. Algunos de los comentarios fueron reiteradamente repetidos por los alumnos han sido en referencia en encontrar un punto clave que indique las partes importantes del tema a tartar y aclaración de conceptos ..."es muy interesante encontrar un documento corto, con las ideas clave y finalización, que aborde de una manera sencilla la introducción de un problema complejo, y ver la utilidad de la material aplicada”..., así mismo otros alumnos comentan ..."lo dificil es introducir un problema complejo, en pocos minutos, ver su utilidad, y que ello me ayude a avanzar por mi mismo en la complejidad de la asignatura. Con los polimedias me ha permitido visionarlos en varias ocasiones y afianzar conceptos para avanzar de manera independiente"....

Se desprende de todas las entrevistas realizadas la buena aceptación de las píldoras de conocimiento por parte de los alumnos.

2- ¿Qué ventajas e inconvenientes consideras que tienen las pildoras en su utilización?.

La totalidad de los alumnos entrevistados consideran en mayor parte las ventajas dadas por el uso de las pildoras de conocimiento, que las desventajas consideradas que en gran medida se las achacan a ellos mismos por su falta de organización, al haber entrado demasiado tarde a visionar los contenidos.

Como ventajas destacan "el tener los conceptos clave en cualquier momento---volver a visionarlos cuando se plantea una duda de conceptos..-el ver al mismo professor que te 
explica las clases magistrales en el video da seguridad para hacer cualquier comentario posterior..--- el tener en pocos minutos de grabación algo que va al grano de lo que queremos conseguir, aunque sea de una manera básica, para poder nosotros avanzar y ampliar posteriormente..., ....mi percepción es que un tema complejo se puede resumir y explicar de una manera sencilla, sabiendo que todo se puede ampliar y mejorar...."

Las desventajas comentadas inciden sobre todo sobre la propia organización del alumno, algunos de los comentarios de los alumnos así lo muestran, aunque la mayoría no observan ninguna desventaja significativa por el uso de las pildoras de aprendizaje. Algunos de los comentarios fueron en esa línea, “....no los había visionado al principio, lo cual fue un error, dado que cuando al final los vi me di cuenta que me aclaraban puntos en los que había perdido excesivo tiempo en entenderlos...”.

Esto confirma la gran aceptación por la mayoría de los alumnos, encontrando de manera mayoriataria ventajas en el uso de las píldoras de conocimiento.

3- ¿Lo ves aplicable a otras asignaturas donde no se hayan realizado pildoras de aprendizaje?.

Al preguntarles a los alumnos si consideran que sería interesante introducer pildoras de conocimiento en otras asignaturas en las que se realize de manera tradicional mediante clases magistrales, la respuestas son unánimes al comentar que ayudaría en gran medida a afianzar conceptos y repasar la materia. Muchos de los comentarios abordaban sobre ello, “....en otras asignaturas, al haber tanta material, se onfunde lo importante con lo intrascendente..., muchas veces no llegamos a entender para que sirve algo, hasta que hemos llegado al final de la asignatura...... ...tenemos que recurrir a tutorias en cuestiones que despues nos damos cuentas que eran obvias, pero no hemos llegado a entender dado que lo importante se confunde en un mar de información...”.

En referencia a esta pregunta, los alumnos consideran que sería muy positive tener pildoras de conocimiento con la información y conceptos relevantes, que ayuden a abordar la asignatura desde el principio, teniendo la libertad ellos mismos de visionarlos cuantas veces quieran si perder excesivos tiempos en tutorias, tal como se hace en las clases convencionales.

4-¿Qué percepción tienes del professor de la asignatura, que ha relizado las pildoras de conocimiento?.

Al visionar en el video (en los polimedias) de la capsula de conocimiento al propio professor que les imparte las clases tradicionales, les da sensación de cercania y seguridad para volver a comentar al professor cualquier duda que se les haya planteado o con posterioridad en el avance de sus estudios.. 
Con lo expuesto se pueden, extraer que utilizar pildoras de conocimiento como apoyo a las clases magistrales en alumnos de máster en materias de arquitectura y edificación, es considerado por ellos como un aliciente importante para mejorar y ampliar sus cnocimientos, así como para recorder en un future conceptos importantes de una determinada asignatura. La libertad de horario para el uso de las pildoras de conocimiento es también muy bien valorada en el sentido de poder elegir ellos mismos el momento del día que quieren accede a ellos, así como las veces que pueden repetirlos. Consideran que ahorran mucho tiempo, dado que aclarar dudas de una manera tradicional conlleva citas, horarios y desplazamientos.

Al ser el estilo de aprendizaje de este tipo de alumnos como reflexive, que se caracteriza por ser estudiantes ponderados, receptivos, analíticos y exhaustivos, observadores, pacientes, cuidadosos y lentos en su proceso de aprendizaje, hace que las pildoras de conocimiento sea una Buena herramienta para mejorar su aprendizaje.

\section{Conclusiones}

Las píldoras de aprendizaje que abordan conceptos básicos e importantes, pueden mejorar la calidad del aprendizaje dado que contribuyen a que los estudiantes no pasen por alto esos conceptos que utilizarán en el futuro a nivel profesional. La ventaja de las píldoras de conocimiento es que los estudiantes pueden disponer de ella siempre que lo necesiten y en cualquier momento y lugar. Con los PoliMedia se consiguen hacer pildoras de aprendizaje por parte del profesor de una manera sencilla, aunque la preparación puede llevar tiempo por parte del professor para intentar sintetizar de una manera simple un problema complejo aunque sea de una manera básica u aproximada.

Los estudiantes de máster en material de arquitectura y edificación, al tener de una manera significativa un estilo de aprendizaje predominante como reflexivo, aceptan de una manera positiva el disponer de recursos formativos en linea para avanzar y afianzar conceptos en el momento de estudio de la asignatura, y con posterioridad cuando quieran recordar conceptos importantes. 


\section{Referencias}

Alonso, C.M.; Gallego, D. y Honey, P. (2005). Los estilos de aprendizaje: Procedimientos de diagnóstico y mejora. Bilbao: Ediciones Mensajero. $6^{\text {a }}$ edición.

Alonso, C.M.; Gallego, D. y Honey, P. (1999). Los estilos de aprendizaje. Procedimientos de Diagnóstico y Mejora. Bilbao: Mensajero.

Alonso, C.M.; Gallego, D.J. y Honey, P. (1994). Los estilos de aprendizaje: Qué son. Cómo diagnosticarlos. Cómo mejorar el propio estilo de aprendizaje. Bilbao: Editorial Mensajero.

Bahamón, J. (2006). El aprendizaje individual permanente: ¿Cómo lograr el desarrollo de esta capacidad de los estudiantes? Cartilla docente, publicaciones del CREA Universidad ICESI.

Bengochea Martínez, L. (2011). Píldoras formativas audiovisuales para el aprendizaje de programación avanzada. Comunicación presentada en las Jornadas de Enseñanza Universitaria de la Informática - JENUI 2011 (pp. 257-263). Sevilla: Asociación de Enseñantes Universitarios de la Informática (AENUI). Disponible en http://hdl.handle.net/2099/11989.

Bengochea, L., \& Budia, F. (2012). Subtitled video tutorials, an accessible teaching material. JACCES - Journal of Accessibility and Design for All, 2(2), 155-164.

Carcel, J; Peñalvo, E. (2016). Construction site managers learning requirements . 4th International Conference on Innovation, Documentation and TeachingTechnologies (INNODOCT 2016). Lean education and innovation. Valencia, Octubre 2016. pp. 313 - 323.

Cárcel Carrasco, F. J.. Roldán Porta, C. (2013). Principios básicos de la Gestión del Conocimiento y su aplicación a la empresa industrial en sus actividades tácticas de mantenimiento y explotación operativa: Un estudio cualitativo. Intangible capital, 9(1), 91-125.

Cárcel Carrasco, F. J., Rodríguez-Méndez, M., Solar, D. A., \& Peñalvo-López, E. (2014a). El estilo de aprendizaje de los egresados en ingenieria de edificación. In Strategies for education in a new context: INNODOCT'14: International Conference on Innovation, Documentation and Teaching Technologies, held on-line in Valencia, Spain, on 8-9 May, 2014. https://riunet. upv. es/handle/10251/40350. (pp. 640-651). Editorial de la Universidad Politécnica de Valencia.

Cárcel Carrasco, F. J., Rodríguez-Méndez, M. (2014b). The style of learning based on the experience of building engineers. 3C Empresa (Vol. 3, No. 2, pp. 69-83). Área de Innovación y Desarrollo, SL.

Carrera, F. (Dir.) (2012). Metodología de las píldoras de conocimiento: Manual del mediador de conocimiento. European Commission: Fundacja Obserwatorium Zarządzania; Prestin Preparação de Estudos e Investimentos, Lda.; ZEUS Consulting S.A.; Instituto Tecnológico de Aragón; and Nowoczesna Firma S.A.

Gallardo Echenique, E. (2015). Aplicación de "píldoras de conocimiento" a través del sistema de poliMedia en el ámbito educativo. XVIII Congreso Internacional Edutec 2015: Educación y tecnología desde una visión transformadora, organizado por la Escuela Superior Politécnica de Chimborazo, Ecuador. Se llevó a cabo los días 17, 18, 19 y 20 de noviembre del 2015. 
Las píldoras de aprendizaje como metodología para afianzar conceptos en alumnos de Máster de Arquitectura y edificación

Gravini, M. (2007). Teoría e investigación de los estilos de aprendizaje. En revista electrónica Diálogos educativos. Año 7, 13, 32-43.

Instituto de Ciencias de la Educación (ICE). (2007). Plan de acciones para la convergencia europea (PACE): "Los objetos de aprendizaje como recurso para la docencia universitaria: criterios para su elaboración." Valencia: Universidad Politécnica de Valencia.

Juanes Méndez, J. A., Prats Galino, A., Álvarez Garrote, H., García Riaza, B., \& Rodríguez Conde, M. J. (2012). Técnicas de borrado digital para la creación de sets virtuales de aprendizaje. CIDUI's Journal.

Disponible http://www.cidui.org/revistacidui/index.php/cidui/article/view/125

Maceiras, R., Cancela, Á., \& Goyanes, V. (2010). Aplicación de Nuevas Tecnologías en la Docencia Universitaria. Formación Universitaria, 3(1), 21-26. doi:10.4067/S0718-50062010000100004

Universitat Politècnica de València (UPV). (2015). Servicios Multimedia: poliMedia. Disponible en https://www.upv.es/entidades/ASIC/catalogo/522359normalc.html 